\title{
Gravitational waves and gamma-ray bursts
}

\author{
Alessandra Corsi ${ }^{1}$ \\ for the LIGO Scientific Collaboration and Virgo collaboration \\ ${ }^{1}$ LIGO laboratory, California Institute of Technology \\ MS 100-36, Pasadena, CA 91125 (USA) \\ email: corsi@caltech.edu
}

\begin{abstract}
Gamma-Ray Bursts are likely associated with a catastrophic energy release in stellar mass objects. Electromagnetic observations provide important, but indirect information on the progenitor. On the other hand, gravitational waves emitted from the central source, carry direct information on its nature. In this context, I give an overview of the multi-messenger study of gamma-ray bursts that can be carried out by using electromagnetic and gravitational wave observations. I also underline the importance of joint electromagnetic and gravitational wave searches, in the absence of a gamma-ray trigger. Finally, I discuss how multi-messenger observations may probe alternative gamma-ray burst progenitor models, such as the magnetar scenario.
\end{abstract}

Keywords. gamma rays: bursts, gravitational waves, stars: neutron, supernovae: general

\section{Introduction}

During the last 15 years, thanks to satellite missions like BeppoSAX (Boella et al. 1997), Swift (Gehrels et al. 2004), and Fermi (Atwood et al. 2009; Meegan et al. 2009), our progress in the understanding of gamma-ray bursts (GRBs) has been quite spectacular. We now know that GRBs are cosmological events related to a catastrophic energy release in stellar mass objects. Energy dissipation within a highly relativistic "fireball", presumably emitted in the form of a jet (e.g., Rhoads 1999; Sari et al. 1999; Kumar \& Panaitescu 2000; Frail et al. 2001), is believed to power the observed $\gamma$-ray flash (prompt emission) and the subsequent "afterglow" (e.g., Blandford \& McKee 1976; Rees \& Mészáros 1992; Mészáros \& Rees 1993a,b; Piran 2004; Mészáros 2006 ).

Traditionally, GRBs have been divided in two main categories, long and short ones (e.g. Kouveliotou et al. 1993), depending on the duration of their prompt $\gamma$-ray emission ( $\lesssim 2 \mathrm{~s}$ for the short bursts, $\gtrsim 2 \mathrm{~s}$ for the long ones). These two populations of bursts are thought to be related to two different progenitor models: "collapsars" for the longsoft bursts (e.g., Woosley 1993; MacFadyen \& Woosley 1999; Piran 2004; Mészáros 2006 ; Woosley \& Bloom 2006, and references therein), and the merger of binary systems of compact objects such as neutron star (NS)-NS or black-hole (BH)-NS systems, for the short-hard ones (e.g., Eichler et al. 1989; Narayan et al. 1992; Janka et al. 1999; Belczynski et al. 2002; Rosswog 2005; Belczynski et al. 2006; Faber et al. 2006).

Despite the recent issues raised in the classification of short and long GRBs based solely on their prompt emission properties (e.g, Zhang et al. 2009), the general picture of two classes of bursts related to two main progenitor models, still holds. The collapsar scenario is observationally supported by the fact that long GRBs occur in galaxies with high specific star formation rate (e.g., Christensen et al. 2004; Castro Cerón et al. 2006; Fruchter et al. 2006; Levesque et al. 2010), and that at least some long GRBs have been observed to be associated with core-collapse supernovae (SNe) of rare type (Galama et al. 1998; Berger et al. 2003; Hjorth et al. 2003; Malesani et al. 2004; Campana et al. 2006; 
Pian et al. 2006). Indeed, the question of what makes some stars die as SNe and some other as relativistic GRBs, is not solved yet (e.g., Woosley \& Bloom 2006, and references therein). Indications that progenitors of short bursts belong, on average, to old stellar populations with a typical lifetime of several Gyr (Barthelmy et al. 2005; Berger et al. 2005; Gehrels et al. 2005; Villasenor et al. 2005; Bloom et al. 2006; Gal-Yam et al. 2008; O'Shaughnessy et al. 2008), provide support to the binary merger scenario.

Collapsars and binary mergers leading to the formation of a $\mathrm{BH}$ plus an accretion disk (e.g., Woosley 1993; Fryer et al. 1996; MacFadyen \& Woosley 1999; Rosswog et al. 1999; Ruffert \& Janka 1999; Narayan et al. 2001), have the potential to power the GRB fireball via the energy released from the accretion of the disk onto the newly formed $\mathrm{BH}$. While the formation of a $\mathrm{BH}$ plus disk system is common to both progenitor models, the more compact scale of the NS-NS (or BH-NS systems), and the less massive debris left over after merger, are invoked to explain the shorter duration and the smaller isotropic energies of these bursts with respect to long ones.

Within the standard fireball model, once the fireball is launched from the central engine, the observed radiation is explained as synchrotron and/or inverse Compton emission from electrons accelerated in internal and external shocks (e.g., Sari 1997; Kobayashi et al. 1997; Sari et al. 1998; Granot et al. 1999; Dermer et al. 2000; Sari \& Esin 2001), taking place at distances $\gtrsim 10^{13} \mathrm{~cm}$ from the central source. High-energy $(\mathrm{GeV})$ tails observed in some GRBs have challenged the internal-external shock fireball model in its simplest formulation (Hurley et al. 1994; Baring \& Harding 1997; Abdo et al. 2009; Kumar \& Barniol Duran 2009; Ackermann et al. 2010; Corsi et al. 2010; De Pasquale et al. 2010; Ghirlanda et al. 2010; Giuliani et al. 2010; Abdo et al. 2011; Asano \& Mészáros 2011; Mészáros \& Rees 2011; Toma et al. 2011; Zhang et al. 2011). However, it remains true that the electromagnetic emission from GRBs, being produced at large distances from the central engine, provides indirect information on the progenitor. On the other hand, gravitational waves (GWs) emitted from the progenitor could directly probe its nature.

\section{GRB-triggered searches for GWs}

Being related to catastrophic events involving stellar-mass objects, GRBs are good candidates for the detection of GWs (Kochanek \& Piran 1993; Finn et al. 1999; van Putten 2001, 2002; Kobayashi \& Mészáros 2003). Coalescing binaries, thought to be associated with short bursts, are one of the most promising GW sources (e.g., Phinney 1991; Cutler et al. 1993; Zhuge et al. 1994; Flanagan \& Hughes 1998; Abadie et al. 2010a; Shibata \& Taniguchi 2011, and references therein) for detectors like the Laser Interferometer Gravitational-Wave Observatory (LIGO; Abbott et al. 2009b) and Virgo (Acernese et al. 2008a; Accadia et al. 2011). For such systems, a chirp signal should be emitted in GWs during the in-spiral, followed by a burst-type signal associated with the merger, and subsequently a signal from the ring-down phase of the newly formed $\mathrm{BH}$ (e.g., ?Kobayashi \& Mészáros 2003; Berti et al. 2009, and references therein). The last, initially deformed, is expected to radiate GWs until reaching a Kerr geometry (Kobayashi \& Mészáros 2003).

In the collapsar scenario, relevant for long GRBs, the high rotation required to form the centrifugally supported disk that powers the GRB, should produce GWs via bar (e.g., Houser et al. 1994; New et al. 2000; Baiotti et al. 2007; Dimmelmeier et al. 2008) or fragmentation instabilities that might develop in the collapsing core (see e.g., Fryer \& New 2003; Ott 2009, for recent reviews) and/or in the disk (Davies et al. 2002; Fryer et al. 2002; Kobayashi \& Mészáros 2003; Piro \& Pfahl 2007). Moreover, asymmetrically 
infalling matter is expected to perturb the final $\mathrm{BH}$ geometry, leading to a ring-down phase (?Kobayashi \& Mészáros 2003).

LIGO and Virgo have been carrying out electromagnetically triggered searches for GWs (Abbott et al. 2005, 2007; Acernese et al. 2007; Abbott et al. 2008b,a; Acernese et al. 2008b; Abbott et al. 2009a; Abadie et al. 2010b; Abbott et al. 2010; Abadie et al. 2011, 2012) over the past decade (for bar detectors electromagnetically triggered searches, see e.g. Astone et al. 1999, 2002, 2005; Baggio et al. 2005). The LIGO Scientific Collaboration operates two LIGO observatories in the U.S. along with the GEO600 detector (Grote \& LIGO Scientific Collaboration 2010) in Germany. Together with Virgo, located in Italy, they form a detector network capable of detecting GW signals arriving from all directions.

GRB-triggered searches for GWs by LIGO and Virgo have targeted both the chirp signal expected in the case of short GRBs during the NS-NS or BH-NS in-spiral, and short unmodeled pulses of GWs that may be expected during the merger/collapse, and ring-down phases of short/long GRBs (Abbott et al. 2005; Acernese et al. 2007; Abbott et al. 2008b,a; Acernese et al. 2008b; Abadie et al. 2010b; Abbott et al. 2010; Abadie et al. 2012). These searches have adopted on-source time windows of few minutes (long GRBs) or few seconds (short GRBs) around the GRB trigger time. In fact, for long GRBs, the time delay between the GW signal and $\gamma$-ray trigger is thought to be dominated by the time necessary for the fireball to push through the stellar envelope of the progenitor (10-100 s; Zhang \& Mészáros 2004). On the other hand, for short GRBs, the NS-NS/BHNS merger is believed to occur quickly, and be over within a few seconds (naturally accounting for the short nature of these bursts). It is estimated that triggered searches for GWs in few minutes time-windows yield a factor of $\approx 2$ improvement in sensitivity with respect to untriggered ones (Kochanek \& Piran 1993).

The most exciting results from LIGO GRB-triggered searches of GWs are probably represented by the cases of the short GRBs 070201 (Abbott et al. 2008a; Ofek et al. 2008) and 051103 (Abadie et al. 2012; Hurley et al. 2010), whose error boxes overlap with nearby galaxies (M31 for GRB 070201; M81 for GRB 051103). A NS-NS binary merger scenario occurring in such hosts was excluded by LIGO with rather high confidence (Abbott et al. 2008a; Abadie et al. 2012). However, the possibility that GRB 070201 and GRB 051103 are related to (extra-galactic) soft gamma-ray repeaters (SGR) giant flares (for a recent review, see ?, and references therein), could not be ruled out. Indeed, LIGO upper-limits for short unmodeled pulses of GWs from GRB 070201 and GRB 051103, are above the maximum GW energy emissible in SGR giant flares (Ioka 2001; Corsi \& Owen 2011).

\section{GW-triggered searches for GRBs}

A very appealing prospect is represented by the possibility of using GWs to trigger electromagnetic (radio, optical, X-ray) follow-ups of GW sources (e.g., ?Bloom et al. 2009; Metzger \& Berger 2012). The discovery of off-axis optical or radio afterglows (Mészáros et al. 1998; Granot et al. 2002; Janka et al. 2006; van Eerten \& MacFadyen 2011) triggered via the (non-beamed) GW emission from the GRB progenitors, would yield a dramatic confirmation of the "jet model", map out the beaming distribution, and provide fundamental inputs to models of relativistic outflows. Radio follow-ups, in particular, are an effective tool to identify relativistic and mildly relativistic outflows (e.g., Kulkarni et al. 1998; Soderberg et al. 2010; Nakar \& Piran 2011) in the absence of a $\gamma$-ray trigger.

Finding electromagnetic counterparts to GW triggers is technically challenging due to imperfect localization of the GW signal and uncertainty regarding the relative 
timing of the GW and electromagnetic emissions. The localization of LIGO-Virgo triplecoincidence GW triggers can yield error-areas of $\sim 100 \mathrm{deg}^{2}$, possibly spread over different patches of the sky (see e.g. Fig. 3 in Abadie et al. 2012). The problem of following-up with optical (or radio, or X-ray) telescopes in such a large error-area can be partially mitigated by: (i) restricting the search for electromagnetic counterparts to transients in nearby galaxies (within the LIGO-Virgo horizon distance); (ii) by noticing that the most promising electromagnetic counterparts of GW events detectable by LIGO and Virgo are expected to be "exotic" (rare) ones (e.g., the orphan afterglow of a GRB, and/or the "kilonova" from a binary merger - see Kulkarni 2005; Metzger et al. 2010).

In 2009-2010, LIGO and Virgo, together with partner electromagnetic observatories, performed their first "LOOC-UP" - Locating and Observing Optical Counterparts to Unmodeled Pulses of gravitational waves - experiment (Kanner et al. 2008; Abadie et al. 2012, and references therein). At the time, there were two operating LIGO interferometers (Abbott et al. 2009b), each with 4-km arms (one near Hanford, Washington, the other in Livingston Parish, Louisiana). The Virgo 3-km arms detector (Acernese et al. 2008a; Accadia et al. 2011) located near Cascina (Italy), was also operating. The LOOC-UP search has established a baseline for low-latency analyses with the next-generation GW detectors (Advanced LIGO and Advanced Virgo; Acernese et al. 2009; Harry \& LIGO Scientific Collaboration 2010). The collaboration between GW and electromagnetic observatories is likely to continue to develop over the next few years, as the scientific community gets ready for a global network of advanced GW detectors.

\section{GRBs and magnetars: prospects for multi-messenger studies}

The forthcoming years may see the development of new GW searches in coincidence with GRBs, aimed at answering some of the questions opened by recent observations. In particular, a compelling result from Swift has been the discovery that the "normal" power-law behavior of long GRB X-ray light curves is often preceded at early times by a steep decay, followed by a shallower-than-normal decay (e.g. Nousek et al. 2006; Zhang et al. 2006). The steep-to-shallow and shallow-to-normal decay transitions are separated by two break times, $100 \mathrm{~s} \lesssim T_{\text {break,1 }} \lesssim 500 \mathrm{~s}$ and $10^{3} \mathrm{~s} \lesssim T_{\text {break,2 }} \lesssim 10^{4} \mathrm{~s}$. It has been suggested that the shallow phase may be attributed to a continuous energy injection by a long-lived central engine, with progressively reduced activity (Zhang et al. 2006).

Newborn magnetars, besides being candidate GRB progenitors (e.g., Usov 1992; Thompson 1994; Bucciantini et al. 2007; Metzger et al. 2007), have also been proposed to account for shallow decays or plateaus observed in GRB light curves (Dai \& Lu 1998; Zhang \& Mészáros 2001; Fan \& Xu 2006; Yu \& Huang 2007; Metzger et al. 2008; Xu et al. 2009; Rowlinson et al. 2010). Independent support for the magnetar scenario comes from the observation of SN 2006aj, associated with the nearby sub-energetic GRB 060218, suggesting that the SN-GRB connection may extend to a much broader range of stellar masses than previously thought, possibly involving two different mechanisms: a "collapsar" for the more massive stars collapsing to a $\mathrm{BH}$, and a newborn (highly-magnetized) NS for the less massive ones (Mazzali et al. 2006).

Several studies have shown how magnetars dipole losses may indeed explain the flattening observed in GRB afterglows (Dai \& Lu 1998; Zhang \& Mészáros 2001; Fan \& Xu 2006; Yu \& Huang 2007; Dall'Osso et al. 2011; Bernardini et al. 2012). Corsi \& Mészáros (2009) have explored a scenario in which the newly born magnetar left over after the GRB explosion undergoes a secular bar-mode instability (Lai \& Shapiro 1995), thus producing a bar-like GW signal associated to the electromagnetic plateau, potentially detectable by the advanced ground-based interferometers like LIGO and Virgo (up to 
distances of $\sim 100 \mathrm{Mpc}$ ). Compared to current analyses that GW detectors are carrying out (see Section 2), this scenario (Corsi \& Mészáros 2009) involves a new class of GW signals, with a longer duration $\left(10^{3}-10^{4} \mathrm{~s}\right)$ and a different frequency evolution. Data analysis techniques for the search of longer duration GW signals possibly applicable to GRB searches, are being developed (e.g., Thrane et al. 2011).

\section{Prospects and conclusions}

The LIGO interferometers are being upgraded to the next-generation Advanced detectors (Harry \& LIGO Scientific Collaboration 2010), that are expected to become operational around 2015. Virgo will also be upgraded to become Advanced Virgo (Acernese et al. 2009). Additionally, the new LCGT detector (Kuroda \& LCGT Collaboration 2010) is being built in Japan. These advanced detectors are expected to detect compact binary coalescences, possibly at a rate of dozens per year after reaching design sensitivity (Abadie et al. 2010a), so that the short-GRB progenitor scenario may finally be probed directly. Long-standing open questions (e.g., is the jet model for GRBs correct? Why do some massive stars die as SNe and others as relativistic GRBs?), or other issues raised by more recent observations (such as the difficulties in the long-short GRB classification; the role of magnetars as GRB progenitors; the link between long GRBs and SGRs, etc.), will greatly benefit from joint GW studies. The advanced GW detectors will provide a totally new view of the transient sky (Márka et al. 2010, 2011). The prospects for this new era of astronomy are exciting, and promise a return of big scientific impact.

\section{Acknowledgments}

LIGO was constructed by the California Institute of Technology and Massachusetts Institute of Technology with funding from the National Science Foundation and operates under cooperative agreement PHY-0757058. This paper has LIGO Document Number LIGO-P1200042.

\section{References}

Abadie, J., et al. 2011, ApJ (Letters), 734, L35

Abadie, J., et al. 2010a, Classical and Quantum Gravity, 27, 173001

Abadie, J., et al. 2010b, ApJ, 715, 1453

Abadie, J., et al. 2012, ArXiv: 1201.4413

Abbott, B., et al. 2005, Phys. Rev. D, 72, 042002

Abbott, B., et al. 2007, Phys. Rev. D., 76, 062003

Abbott, B., et al. 2008a, ApJ, 681, 1419

Abbott, B., et al. 2008b, Phys. Rev. D, 77, 062004

Abbott, B. P., et al. 2010, ApJ, 715, 1438

Abbott, B. P., et al. 2009a, Reports on Progress in Physics, 72, 076901

Abbott, B. P., et al. 2009b, ApJ (Letters), 701, L68

Abdo, A. A., et al. 2011, ApJ (Letters), 734, L27

Abdo, A. A., et al. 2009,Science, 323, 1688

Accadia, T., et al. 2011, Classical and Quantum Gravity, 28, 114002

Acernese, F., et al. 2009, Note VIR-027A09

Acernese, F., et al. 2008a, Classical and Quantum Gravity, 25, 184001

Acernese, F., et al. 2008b, Classical and Quantum Gravity, 25, 225001

Acernese, F., et al. 2007, Classical and Quantum Gravity, 24, 671

Ackermann, M., et al. 2010, ApJ, 716, 1178

Asano, K. \& Mészáros, P. 2011, ApJ, 739, 103

Astone, P., et al. 2005, Phys. Rev. D, 71, 042001

Astone, P., et al. 1999, Astroparticle Physics, 10, 83

Astone, P., et al. 2002, Phys. Rev. D, 66, 102002 
Atwood, W. B., et al. 2009, ApJ, 697, 1071

Baggio, L., et al. 2005, Physical Review Letters, 95, 081103

Baiotti, L., de Pietri, R., Manca, G. M., \& Rezzolla, L. 2007, Phys. Rev. D, 75, 044023

Baring, M. G. \& Harding, A. K. 1997, ApJ, 491, 663

Barthelmy, S. D., et al. 2005, Nature, 438, 994

Belczynski, K., Bulik, T., \& Rudak, B. 2002, ApJ, 571, 394

Belczynski, K., Perna, R., Bulik, T., Kalogera, V., Ivanova, N., \& Lamb, D. Q. 2006, ApJ, 648, 1110

Berger, E., et al. 2003, Nature, 426, 154

Berger, E., et al. 2005, Nature, 438, 988

Bernardini, M. G., Margutti, R., Mao, J., Zaninoni, E., \& Chincarini, G. 2012, A\&SA, 539, A3

Berti, E., Cardoso, V., \& Starinets, A. O. 2009, Classical and Quantum Gravity, 26, 163001

Blandford, R. D. \& McKee, C. F. 1976, Physics of Fluids, 19, 1130

Bloom, J. S., et al. 2009, ArXiv: 0902.1527

Bloom, J. S., et al. 2006, ApJ, 638, 354

Boella, G., Butler, R. C., Perola, G. C., Piro, L., Scarsi, L., \& Bleeker, J. A. M. 1997, A\&ऽAS, 122,299

Bouhou, B. \& for the ANTARES Collaboration, the LIGO Scientific Collaboration, the Virgo Collaboration. 2012, ArXiv: 1201.2840

Bucciantini, N., Quataert, E., Arons, J., Metzger, B. D., \& Thompson, T. A. 2007, MNRAS, 380,1541

Campana, S., et al. 2006, Nature, 442, 1008

Castro Cerón, J. M., Michałowski, M. J., Hjorth, J., Watson, D., Fynbo, J. P. U., \& Gorosabel, J. 2006, ApJ (Letters), 653, L85

Christensen, L., Hjorth, J., \& Gorosabel, J. 2004, A\&A A, 425, 913

Corsi, A., Guetta, D., \& Piro, L. 2010, ApJ, 720, 1008

Corsi, A. \& Mészáros, P. 2009, ApJ, 702, 1171

Corsi, A. \& Owen, B. J. 2011, Phys. Rev. D, 83, 104014

Cutler, C., et al. 1993, Physical Review Letters, 70, 2984

Dai, Z. G. \& Lu, T. 1998, A\& A, 333, L87

Dall'Osso, S., Stratta, G., Guetta, D., Covino, S., de Cesare, G., \& Stella, L. 2011, A\&A, 526, A121

Davies, M. B., King, A., Rosswog, S., \& Wynn, G. 2002, ApJ (Letters), 579, L63

De Pasquale, M., et al. 2010, ApJ (Letters), 709, L146

Dermer, C. D., Chiang, J., \& Mitman, K. E. 2000, ApJ, 537, 785

Dimmelmeier, H., Ott, C. D., Marek, A., \& Janka, H.-T. 2008, Phys. Rev. D, 78, 064056

Echeverria, F. 1989, Phys. Rev. D, 40, 3194

Eichler, D., Livio, M., Piran, T., \& Schramm, D. N. 1989, Nature, 340, 126

Faber, J. A., Baumgarte, T. W., Shapiro, S. L., \& Taniguchi, K. 2006, ApJ (Letters), 641, L93

Fan, Y. \& Xu, D. 2006, MNRAS, 372, L19

Finn, L. S., Mohanty, S. D., \& Romano, J. D. 1999, Phys. Rev. D, 60, 121101

Flanagan, É. É. \& Hughes, S. A. 1998, Phys. Rev. D, 57, 4535

Frail, D. A., et al. 2001, ApJ (Letters), 562, L55

Fruchter, A. S., et al. 2006, Nature, 441, 463

Fryer, C. L., Benz, W., \& Herant, M. 1996, ApJ, 460, 801

Fryer, C. L., Holz, D. E., \& Hughes, S. A. 2002, ApJ, 565, 430

Fryer, C. L. \& New, K. C. 2003, Living Reviews in Relativity, 6

Gal-Yam, A., et al. 2008, ApJ, 686, 408

Galama, T. J., et al. 1998, Nature, 395, 670

Gehrels, N., et al. 2004, ApJ, 611, 1005

Gehrels, N., et al. 2005, Nature, 437, 851

Ghirlanda, G., Ghisellini, G., \& Nava, L. 2010, A\&A, 510, L7

Giuliani, A., et al. 2010, ApJ (Letters), 708, L84

Granot, J., Panaitescu, A., Kumar, P., \& Woosley, S. E. 2002, ApJ (Letters), 570, L61 
Granot, J., Piran, T., \& Sari, R. 1999, ApJ, 527, 236

Grote, H., \& LIGO Scientific Collaboration. 2010, Classical and Quantum Gravity, 27, 084003

Harry, G. M., \& LIGO Scientific Collaboration. 2010, Classical and Quantum Gravity, 27, 084006 Hjorth, J., et al. 2003, Nature, 423, 847

Houser, J. L., Centrella, J. M., \& Smith, S. C. 1994, Physical Review Letters, 72, 1314

Hurley, K., et al. 1994, Nature, 372, 652

Hurley, K., et al. 2010, MNRAS, 403, 342

Ioka, K. 2001, MNRAS, 327, 639

Janka, H.-T., Aloy, M.-A., Mazzali, P. A., \& Pian, E. 2006, ApJ, 645, 1305

Janka, H.-T., Eberl, T., Ruffert, M., \& Fryer, C. L. 1999, ApJ (Letters), 527, L39

Kanner, J., Huard, T. L., Márka, S., Murphy, D. C., Piscionere, J., Reed, M., \& Shawhan, P. 2008, Classical and Quantum Gravity, 25, 184034

Kobayashi, S. \& Mészáros, P. 2003, ApJ, 589, 861

Kobayashi, S., Piran, T., \& Sari, R. 1997, Apj, 490, 92

Kochanek, C. S. \& Piran, T. 1993, ApJ (Letters), 417, L17

Kouveliotou, C., Meegan, C. A., Fishman, G. J., Bhat, N. P., Briggs, M. S., Koshut, T. M., Paciesas, W. S., \& Pendleton, G. N. 1993, ApJ (Letters), 413, L101

Kulkarni, S. R., et al. 1998, Nature, 395, 663

Kulkarni, S. R. 2005, ArXiv: astro-ph/0510256

Kumar, P. \& Barniol Duran, R. 2009, MNRAS, 400, L75

Kumar, P. \& Panaitescu, A. 2000, ApJ (Letters), 541, L9

Kuroda, K., \& LCGT Collaboration. 2010, Classical and Quantum Gravity, 27, 084004

Lai, D. \& Shapiro, S. L. 1995, ApJ, 442, 259

Levesque, E. M., Berger, E., Kewley, L. J., \& Bagley, M. M. 2010, AJ, 139, 694

MacFadyen, A. I. \& Woosley, S. E. 1999, ApJ, 524, 262

Malesani, D., et al. 2004, ApJ (Letters), 609, L5

Márka, S. \& for LIGO Scientific Collaboration, Virgo Collaboration. 2011, Classical and Quantum Gravity, 28, 114013

Márka, S. \& LIGO Scientific Collaboration, Virgo Collaboration. 2010, Journal of Physics Conference Series, 243, 012001

Mazzali, P. A., et al. 2006, Nature, 442, 1018

Meegan, C., et al. 2009, ApJ, 702, 791

Mereghetti, S. 2008, A\&SARv, 15, 225

Mészáros, P. 2006, Reports on Progress in Physics, 69, 2259

Mészáros, P. \& Rees, M. J. 1993a, ApJ (Letters), 418, L59

Mészáros, P. \& Rees, M. J. 1993b, ApJ, 405, 278

Mészáros, P. \& Rees, M. J. 2011, ApJ (Letters), 733, L40

Mészáros, P., Rees, M. J., \& Wijers, R. A. M. J. 1998, ApJ, 499, 301

Metzger, B. D. \& Berger, E. 2012, ApJ, 746, 48

Metzger, B. D., et al. 2010, MNRAS, 406, 2650

Metzger, B. D., Quataert, E., \& Thompson, T. A. 2008, MNRAS, 385, 1455

Metzger, B. D., Thompson, T. A., \& Quataert, E. 2007, ApJ, 659, 561

Nakar, E. \& Piran, T. 2011, Nature, 478, 82

Narayan, R., Paczynski, B., \& Piran, T. 1992, ApJ (Letters), 395, L83

Narayan, R., Piran, T., \& Kumar, P. 2001, ApJ, 557, 949

New, K. C. B., Centrella, J. M., \& Tohline, J. E. 2000, Phys. Rev. D, 62, 064019

Nousek, J. A., et al. 2006, ApJ, 642, 389

Ofek, E. O., et al. 2008, ApJ, 681, 1464

O'Shaughnessy, R., Belczynski, K., \& Kalogera, V. 2008, ApJ, 675, 566

Ott, C. D. 2009, Classical and Quantum Gravity, 26, 063001

Phinney, E. S. 1991, ApJ (Letters), 380, L17

Pian, E., et al. 2006, Nature, 442, 1011

Piran, T. 2004, Reviews of Modern Physics, 76, 1143

Piro, A. L. \& Pfahl, E. 2007, ApJ, 658, 1173 
Rau, A. et al. 2009, PASP, 121, 1334

Rees, M. J. \& Mészáros, P. 1992, MNRAS, 258, 41P

Rhoads, J. E. 1999, ApJ, 525, 737

Rosswog, S. 2005, ApJ, 634, 1202

Rosswog, S., Liebendörfer, M., Thielemann, F.-K., Davies, M. B., Benz, W., \& Piran, T. 1999, $A \mathscr{G} A, 341,499$

Rowlinson, A., et al. 2010, MNRAS, 409, 531

Ruffert, M. \& Janka, H.-T. 1999, A\&A, 344, 573

Sari, R. 1997, ApJ (Letters), 489, L37

Sari, R. \& Esin, A. A. 2001, ApJ, 548, 787

Sari, R., Piran, T., \& Halpern, J. P. 1999, ApJ (Letters), 519, L17

Sari, R., Piran, T., \& Narayan, R. 1998, ApJ (Letters), 497, L17

Shibata, M. \& Taniguchi, K. 2011, Living Reviews in Relativity, 14, 6

Soderberg, A. M., et. al. 2010, Nature, 463, 513

Sylvestre, J. 2003, ApJ, 591, 1152

Thompson, C. 1994, MNRAS, 270, 480

Thrane, E., et al. 2011, Phys. Rev. D, 83, 083004

Toma, K., Wu, X.-F., \& Mészáros, P. 2011, MNRAS, 415, 1663

Usov, V. V. 1992, Nature, 357, 472

van Eerten, H. J. \& MacFadyen, A. I. 2011, ApJ (Letters), 733, L37

van Putten, M. H. P. M. 2001, ApJ (Letters), 562, L51

van Putten, M. H. P. M. 2002, ApJ (Letters), 575, L71

Villasenor, J. S., et al. 2005, Nature, 437, 855

Woosley, S. E. 1993, ApJ, 405, 273

Woosley, S. E. \& Bloom, J. S. 2006, ARA\&A, 44, 507

Xu, M., Huang, Y., \& Lu, T. 2009, Research in Astronomy and Astrophysics, 9, 1317

Yu, Y. \& Huang, Y. 2007, Chinese Journal of Astronomy and Astrophysics, 7, 669

Zhang, B., Fan, Y. Z., Dyks, J., Kobayashi, S., Mészáros, P., Burrows, D. N., Nousek, J. A., \& Gehrels, N. 2006, ApJ, 642, 354

Zhang, B. \& Mészáros, P. 2001, ApJ (Letters), 552, L35

Zhang, B. \& Mészáros, P. 2004, International Journal of Modern Physics A, 19, 2385

Zhang, B., et al. 2009, ApJ, 703, 1696

Zhang, B.-B., et al. 2011, ApJ, 730, 141

Zhuge, X., Centrella, J. M., \& McMillan, S. L. W. 1994, Phys. Rev. D, 50, 6247

Discussion

ASTRAATMADJA: Is the angular resolution of the GW detectors good enough to search for an electromagnetic counterpart? Do you also intend to look for neutrino signals?

CORSI: The error-area for triple coincidence GW events from the LIGO-Virgo network is $\sim 100$ $\operatorname{deg}^{2}$, much bigger than e.g. the $\approx 2^{\prime \prime}$ FWHM of a telescope like the Palomar 48-inch (Rau et al. 2009). While a large number of optical transients is expected to be found in the GW errorarea, the problem can be mitigated by selecting only the most promising for a GW detection (in nearby galaxies and likely of "exotic", rare type). Joint searches for GWs and high energy neutrinos (though, currently, not specifically within the LOOC-UP experiment) are indeed being performed (see e.g., Bouhou et al. 2012, and references therein).

METZGER: In the magnetar scenario proposed for explaining GRB plateaus, can sufficiently rapid rotation be maintained in the presence of enhanced early spin-down by neutrino emission?

CORSI: Sufficiently high rotation should be maintained to explain the observed plateaus: typically, a $(1-5)$ ms magnetar with $B \sim(1-10) \times 10^{14} \mathrm{G}$ is required from modeling of the X-ray light curves with plateaus (e.g., Zhang \& Mészáros 2001; Yu \& Huang 2007; Xu et al. 2009; Dall'Osso et al. 2011). As you have shown (Metzger et al. 2007), enhanced spin-down by neutrino emission at earlier timescales may be an issue, but likely only for the shortest periods and highest magnetic fields in these ranges. 